\title{
MAPA DE VULNERABILIDAD A LA CONTAMINACIÓN ACUÍFERA, CANTÓN DE BELÉN, HEREDIA, COSTA RICA
}

\author{
GROUNDWATER POLLUTION HAZARD MAP, BELEN COUNTY, \\ HEREDIA, COSTA RICA
}

\author{
Roberto Protti* \& Dennis Sojo \\ Geotest S.A. Apdo. 1291-2050, San Pedro, San José \\ *Autor para contacto: rprotti@geotestcr.com
}

(Recibido: 12/09/2014; aceptado: /8/09/2015)

\begin{abstract}
GOD methodology (Foster et al., 2002) was applied to produce a Groundwater pollution vulnerability map for the Belen County, Heredia, Costa Rica. This map was prepared during 2013-2014 as part of the hydro geological investigations done by the author in the area of Belen. A new vulnerability classification is proposed related to the official map available by Belen Municipality.

Keywords: Barba, Colima, aquifer, vulnerability, Belen, hydrogeology.

RESUMEN: Mediante la aplicación de la metodología GOD (Foster et al., 2002), se elaboró un mapa de vulnerabilidad a la contaminación acuífera para el cantón de Belén, Heredia, Costa Rica. Este mapa fue elaborado durante entre los años 2013 y 2014 como parte de las investigaciones hidrogeológicas realizadas por los autores en el cantón de Belén. Se propone una nueva clasificación de la vulnerabilidad a la contaminación acuífera con respecto a los mapas vigentes actualmente por parte de la Municipalidad de Belén.

Palabras clave: Barba, Colima, acuífero, vulnerabilidad, Belén, hidrogeología.
\end{abstract}




\section{INTRODUCCIÓN}

Entre los años 2009 y 2010, y a solicitud de la Municipalidad de Belén, la Universidad de Costa Rica (UCR-Fundevi) y el Servicio nacional de Aguas Subterráneas Riego y Avenamiento (SENARA) produjeron, inspeccionaron y aprobaron respectivamente un estudio hidrogeológico cuyo objetivo básico era determinar la dimensión de las zonas de protección de los pozos de abastecimiento público propiedad de la municipalidad de Belén (Vargas, 2009).

Uno de los productos de ese estudio fue el mapa de vulnerabilidad a la contaminación acuífera del cantón, mapa que, debido a la legislación actual, fue impuesto como vinculante y de acatamiento obligatorio a la Municipalidad de Belén para la regulación del uso del suelo. Sin embargo, entre los años 2013 y 2015, los autores de esta investigación y otros hidrogeólogos realizaron más de 20 estudios de verificación de la clasificación de vulnerabilidad dada en el mapa oficial, con el resultado de que se ha verificado indudablemente, que la clasificación oficial no corresponde con las condiciones hidrogeológicas reales del cantón.

De esta forma, a solicitud de un grupo importante de vecinos de Belén, y con el objetivo de producir un mapa más realista de todo el cantón, los autores elaboraron un nuevo mapa que comprende toda el área cantonal de Belén, el cual se expone en este artículo, con el objetivo de suministrar al municipio local un instrumento de regulación más acorde con la realidad hidrogeológica local. Aunque este mapa no es oficial ya que carece de la aprobación por parte de Senara, se ha demostrado abundante e indudablemente (mediante estudios específicos revisados y aprobados por SENARA) que el mapa propuesto representa en forma más realista, la situación hidrogeológica de ese cantón.

\section{METODOLOGÍA}

Esta investigación se realizó mediante la aplicación de la siguiente secuencia metodológica:

- Elaboración de mapa base de trabajo sobre la base topográfica "San Antonio" a escala 1:10 000 digitalizada para efectos de georeferenciación de campo.
- Recopilación de la información hidrogeológica disponible sobre el área en cuestión, en particular de los datos estratigráficos contenidos en los archivos oficiales de perforaciones (SENARA).

- Levantamiento detallado de campo con referenciación mediante GPS de afloramientos y demás elementos necesarios para la elaboración de los mapas geológico e hidrogeológico del área.

- Perforación y análisis de datos de 6 sondeos estratigráficos para instalación de piezómetros realizadas mediante rotación al diamante, método penetración estándar (SPT) y penetración de cono dinámico (DCPT) por la firma Geotest S.A., entre mayo y setiembre del año 2013 en sitios distribuidos dentro de las zonas clasificadas como de vulnerabilidad extrema en estudios previos.

- Análisis de los datos obtenidos para determinar los parámetros hidrogeológicos básicos que intervienen en las zonificaciones de vulnerabilidad a la contaminación acuífera. Elaboración de mapas de las variables G, O y D.

- Superposición de los mapas paramétricos para la elaboración del mapa final de vulnerabilidad a la contaminación acuífera específico para el acuífero Barba.

A pesar de la existencia de varios métodos para la evaluación de la vulnerabilidad a la contaminación de acuíferos (Auge, 2004), y con el fin de mantener la misma metodología utilizada en la elaboración de los mapas oficiales y actualmente vigentes, se aplicó en esta investigación el denominado método G.O.D. (Foster et al., 2002), cuyos parámetros y diagrama de flujo de valoraciones se muestra en la gráfica de la figura 1.

El método en cuestión se basa en la determinación del Índice de Vulnerabilidad Acuífera (I.V.A.) mediante la asignación de valores a los rangos de variación de tres parámetros controladores a saber:

- Parámetro G: se refiere a la categorización del tipo de acuífero presente en el subsuelo. En este caso se asignan valores que varían entre $0 \mathrm{y}$ 1,0 dependiendo del tipo de acuífero, que puede variar entre confinado, semiconfinado, no confinado cubierto y no confinado sin coberturas.

- Parámetro O: se refiere a la litología de los materiales que cubren al acuífero en estudio, a su carácter de consolidación, y al tipo 


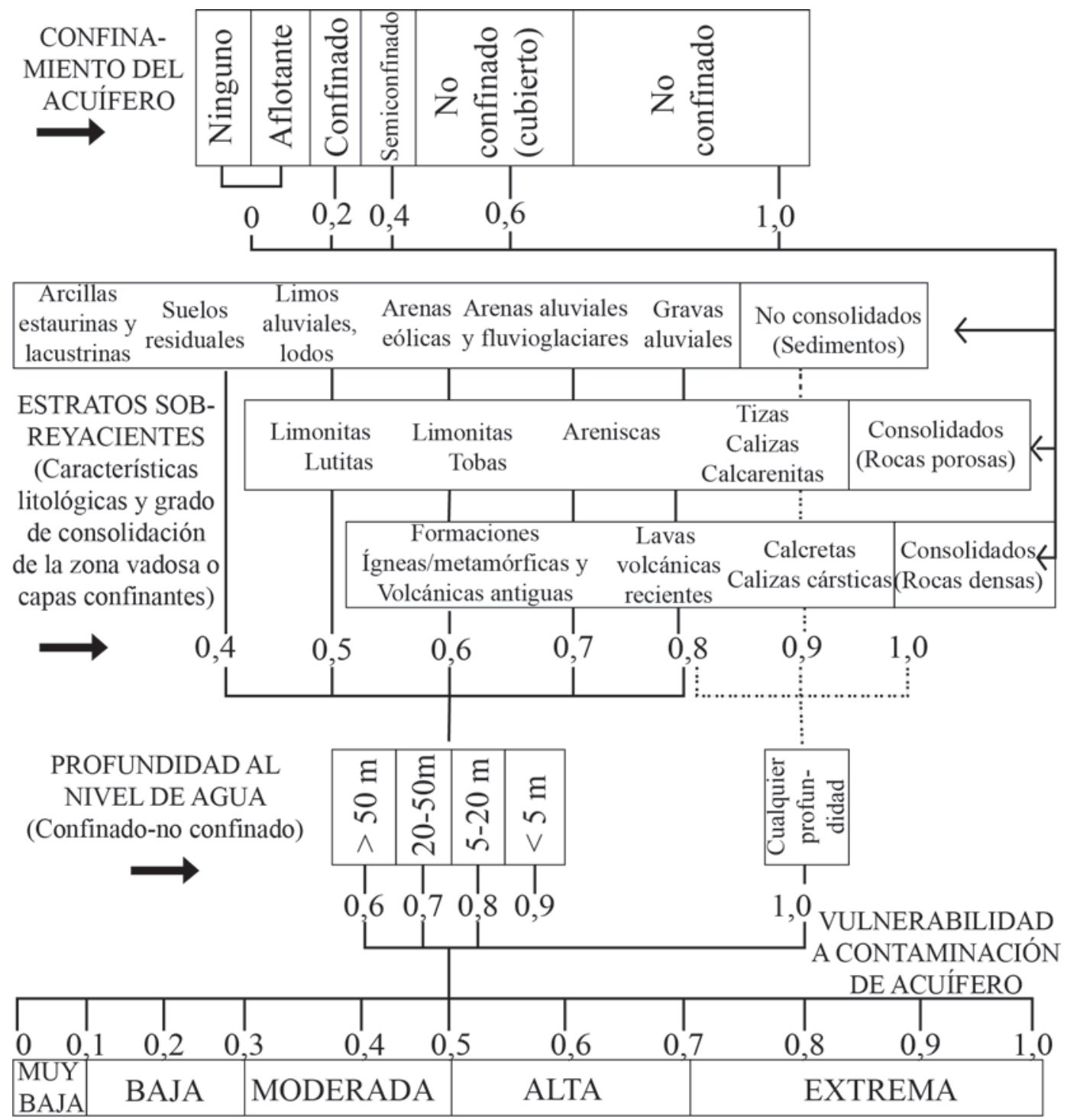

Fig. 1: Gráfica del método G.O.D. para definir la vulnerabilidad a la contaminación acuífera (fuente: Foster, et al. [2002]).

de roca o materiales que lo componen, es decir, a la litología y características de los materiales a través de los que ocurre la infiltración en la zona no saturada hasta alcanzar el nivel de saturación. Dependiendo del tipo de coberturas se asignan valores de entre 0,4 y 1,0 a este parámetro.

- Parámetro D: se refiere a la profundidad o rango de profundidad al nivel de saturación, es decir, considera el espesor de la zona no saturada sobre el nivel de saturación en cuestión. Este parámetro varía entre 0,6 para profundidad mayor que $50 \mathrm{~m}$ y 0,9 para profundidad menor que $5 \mathrm{~m}$.

De acuerdo con lo anterior, los trabajos realizados durante esta investigación se concentraron en definir a escala de detalle, los valores (y sus variaciones) de los parámetros que definen la vulnerabilidad a la contaminación acuífera dentro del área estudiada, es decir, hacia la elaboración de mapas detallados de los tipos de acuífero presentes en el subsuelo (Parámetro G), de la presencia o no de 
coberturas sobre estos, y en caso afirmativo, del tipo litológico y clasificación de consolidación de las coberturas (Parámetro O), y de la profundidad al nivel de saturación a la mejor escala posible (Parámetro D).

Dado que el parámetro D es crítico para definir si una zona es o no de vulnerabilidad extrema, las perforaciones realizadas durante esta investigación se concentraron en definir si existe o no en los sitios de perforación un nivel de saturación a menos de $5 \mathrm{~m}$ de profundidad, y si los materiales de las coberturas superficiales son o no arcillosos.

\section{ESTUDIO DE VULNERABILIDAD A LA CONTAMINACIÓN ACUÍFERA}

\section{Estratigrafía de acuíferos y parámetros de zonificación G.O.D.}

El cantón de Belén, forma parte del denominado Campo Volcánico Central de Costa Rica, el cual se compone de rocas y materiales de origen volcánico de edad Cuaternario.

La base estratigráfica conocida de esta región (Fig. 2) está compuesta por una espesa secuencia de lavas diaclasadas, brechas lávicas de alta permeabilidad, y algunas capas de ignimbritas intercaladas, que se han correlacionado con las unidades acuíferas de Colima inferior (Miembro Belén), Unidad Puente de Mulas y unidad Colima superior (Miembro Linda Vista) (SENARA-BGS, 1985).

Las unidades acuíferas de Colima son de tipo confinado a semiconfinado, y únicamente se observan afloramientos de estas rocas a lo largo del cañón del río Virilla en el extremo Sur del cantón de Belén. La recarga de los acuíferos de Colima no ocurre dentro del cantón de Belén.

Los acuíferos de Colima han sido identificados en perforaciones de pozos en este cantón a profundidades usualmente mayores que $50 \mathrm{~m}$ bajo el nivel de superficie.

Los acuíferos de Colima se encuentran cubiertos y confinados por espesas capas de tobas soldadas e ignimbritas, correlacionadas con la Unidad acuitardo/acuicierre Tiribi, cuya permeabilidad es menor que $\mathrm{k}<0,00027 \mathrm{~m} / \mathrm{d}$ (Ramírez \& Alfaro, 2002), es decir, se trata de materiales

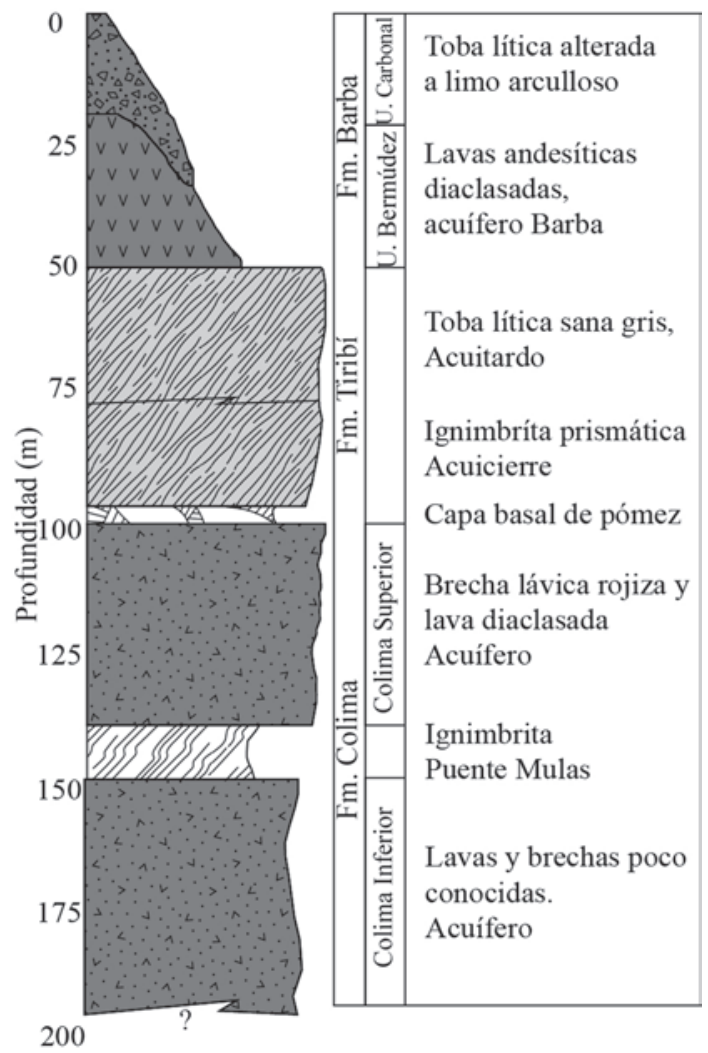

Fig. 2: Columna estratigráfica e hidrogeológica del cantón de Belén

prácticamente impermeables o de muy baja permeabilidad que actúan como capa cierre que inhibe la percolación de aguas desde la superficie hasta el nivel de saturación de dichos acuíferos.

Las ignimbritas de la unidad Tiribi se emplazaron como flujos piroclásticas en una aparentemente rápida sucesión de eventos volcánicos explosivos ocurrida hace unos 300 Ka. (Pérez, et al., 2006).

Las rocas del acuicierre Tiribi tienen un espesor medio que supera los $30 \mathrm{~m}$, y es posible observar afloramientos de este tipo de rocas a lo largo de los taludes del cañón del río Virilla y en algunas de las áreas bajas del sector central y Oeste del cantón (Fig. 3A).

Sobre las rocas de la unidad Tiribi se presentan rocas de la Formación Barba, cuyo miembro inferior está conformado por lavas andesíticas diaclasadas denominadas Unidad Bermúdez, y su miembro superior está compuesto por coberturas 


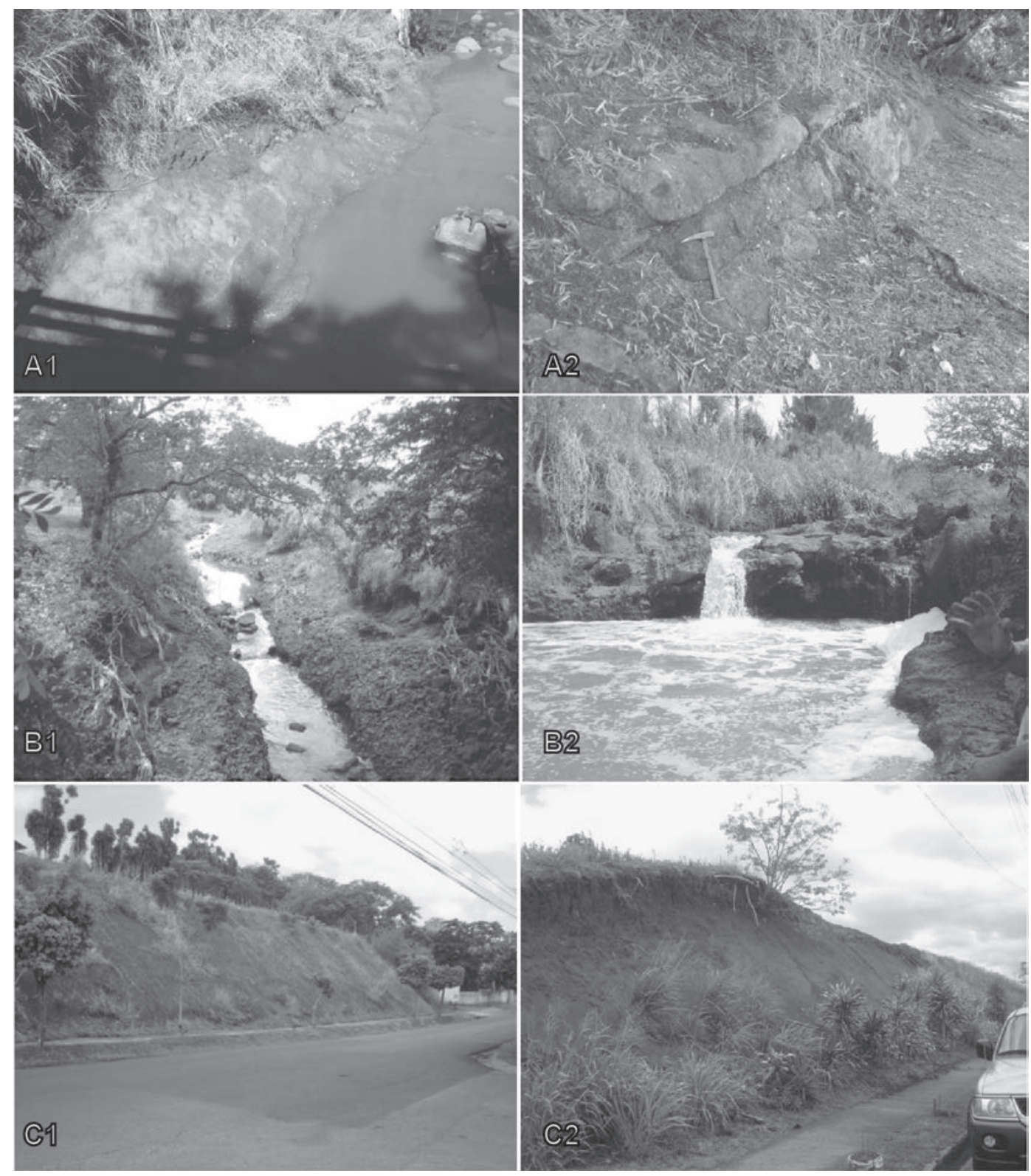

Fig. 3: A1) Ignimbritas de la Fm. Tiribí en el lecho del río Bermúdez (217613-518406). A2) Talud de calle interna, Residencial Cariari (217617-518705). B1) Techo de la unidad Bermúdez en el cauce del río Burío (219000-518803. Nótese la cobertura de toba alterada). B2) Frente terminal de unidad Bermúdez en lecho del río Burío (218835-516649). C1 y C2) Zonas donde la unidad Bermúdez está cubierta por espesas coberturas de toba alterada de la unidad Carbonal (219238-515573 y 219592-515458 respectivamente).

de tobas de ceniza alterada a limo arcilloso de la Unidad Carbonal (Protti, 1986).

La Unidad "acuífero Barba" contiene un acuífero con flujo de agua subterránea de tipo fisural dentro de las capas de lavas diaclasadas de la Unidad Bermúdez, y se compone de al menos dos coladas individuales de lavas andesíticas cuya edad es menor que $300 \mathrm{Ka}$.

Las lavas de la Unidad Bermúdez dan origen al acuífero Barba, de tipo freático (no confinado) 
con coberturas de baja permeabilidad de tobas volcánicas alteradas de la Unidad Carbonal. Estas rocas afloran, es decir, se encuentran en superficie y sin coberturas, en forma dispersa a lo largo de los cauces de los ríos Segundo y Burío, y en los escarpes del frente terminal de las coladas de lava. (Fig. 3B)

La estratigrafía de unidades acuíferas presentes en el cantón de Belén se resume en la columna estratigráfica de la figura 2.

La figura 1 permite establecer por un lado que el acuífero confinado de Colima superior se encuentra cubierto por unidades de tipo acuitardo/ acuicierre (Fm. Tiribi) de tal forma que su vulnerabilidad a la contaminación es en todos los casos baja (I.V.A. = G x O x D = 0,4 x 0,6 x 1 = 0,24), salvo en donde las rocas de esta unidad afloran en superficie en algunos sitios del cañón del río Virilla y en el piso de canteras ubicadas junto a este, y por otro, que el acuífero superficial Barba tiene vulnerabilidad que depende de si el acuífero aflora o no, del tipo de coberturas sobre este cuando están presentes, y de la profundidad al nivel de saturación.

Así, se definió que existen en el cantón de Belén dos casos concretos de zonas de vulnerabilidad con respecto al acuífero Barba, según se muestra en la figura 4a y 4b (método GOD específico para el área estudiada) a saber:

Caso A: zonas en donde las unidades de lava del acuífero Barba (Unidad Bermúdez) se encuentran cubiertas por tobas alteradas de la unidad Carbonal. En este caso, para el tipo de acuífero en cuestión (no confinado (Ramírez \& Alfaro, 2002) cubierto), el valor $\mathrm{G}$ correspondiente es de $\mathrm{G}=0,6$. Las coberturas de tobas volcánicas de acuerdo con el método de análisis, corresponden con un valor $\mathrm{O}=0$,6 (Fig. 4a).

De esta forma, para las zonas en donde el acuífero Barba se encuentra cubierto por tobas de la unidad Carbonal, el índice de vulnerabilidad a la contaminación puede tener valor máximo de IVA $=0,6 \times 0,6 \times 1=0,36$ para zonas con el nivel de saturación a menos de $2 \mathrm{~m}$ de profundidad $(\mathrm{D}=1)$, y mínimo de IVA $=0,6 \times 0,6 \times 0,6=0,21$ para las zonas en donde el nivel de saturación se localiza a más de $20 \mathrm{~m}$ de profundidad $(\mathrm{D}=0,6$.).

El rango de variación del IVA para el caso A indica que las zonas en donde el acuífero Barba se encuentra cubierto por tobas alteradas de la unidad Carbonal, solo pueden clasificarse como de vulnerabilidad de baja a moderada dependiendo de la profundidad al nivel de saturación, y en ningún caso como de vulnerabilidad alta o extrema (Fig. 3C y 4a).

Caso B: Zonas en donde el acuífero Barba aflora en superficie (ver figura $5 \mathrm{~A}$ ), es decir, sin coberturas de tobas alteradas o con delgadas coberturas de depósitos aluviales de granulometría fina. En este caso, para el tipo de acuífero, es decir, no confinado, el valor correspondiente al parámetro es $G=1$. Donde la percolación de aguas en la zona no saturada hasta el nivel de saturación ocurre a través de lavas (Unidad Bermúdez), el valor del parámetro es de $\mathrm{O}=0,8$.

El valor del IVA para el acuífero Barba en las zonas en donde este aflora sin coberturas varía entre un máximo de IVA $=1 \times 0,8 \times 1=0,8$ para las zonas en donde el nivel de saturación se localiza a menos de $2 \mathrm{~m}$ de profundidad $(\mathrm{D}=1)$ y un mínimo de IVA $=1 \times 0,8 \times 0,6=0,48$ para las zonas en donde el nivel de saturación se localiza a más de $20 \mathrm{~m}$ de profundidad.

Así, las zonas en donde el acuífero Barba aflora en superficie pueden ser clasificadas como de vulnerabilidad moderada si el nivel de saturación se ubica a más de $20 \mathrm{~m}$ de profundidad $(\mathrm{D}=0,6)$, vulnerabilidad alta si el nivel de saturación se ubica entre 5 y $20 \mathrm{~m}$ de profundidad $(D=0,7$ a 0,8$)$, y únicamente como de vulnerabilidad extrema si el nivel de saturación se localiza a menos de $5 \mathrm{~m}$ de profundidad ( $\mathrm{D}=0,9$ a 1$)$.

Para clasificar como extrema la vulnerabilidad a la contaminación del acuífero Barba, es necesario pero no suficiente que las lavas de la unidad Bermúdez afloren en superficie, también se requiere que el nivel de saturación se localice a menos de $5 \mathrm{~m}$ de profundidad, y es con este objetivo (definir si el nivel de saturación se ubica a menos de $5 \mathrm{~m}$ en las zonas definidas como de vulnerabilidad extrema en el mapa oficial) que se realizó la campaña de perforaciones entre mayo y setiembre del año 2013.

De acuerdo con el modelo de la figura 4b, para las condiciones geológicas e hidrogeológicas del acuífero Barba en el cantón de Belén, no es posible numéricamente que exista contacto entre zonas de vulnerabilidad extrema y vulnerabilidad 


\begin{tabular}{|c|c|c|c|c|}
\hline \multicolumn{5}{|c|}{$\begin{array}{l}\text { I.V.A. = G x O x D Índice de } \\
\text { vulnerabilidad acuífera CASO A }\end{array}$} \\
\hline $\begin{array}{l}\mathrm{G}^{*} \\
\text { Tipo } \\
\text { Acu- } \\
\text { ifero }\end{array}$ & \begin{tabular}{|c}
$\mathrm{O}^{*}$ \\
Tipo de \\
cobe- \\
rtura
\end{tabular} & $\begin{array}{c}\text { D** } \\
\text { Profundidad } \\
\text { a nivel de } \\
\text { saturación } \\
\end{array}$ & $\mathrm{GxOxD}$ & $\begin{array}{l}\text { Clasifi- } \\
\text { cación }\end{array}$ \\
\hline 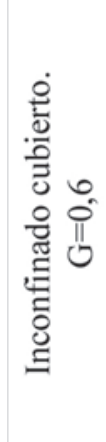 & 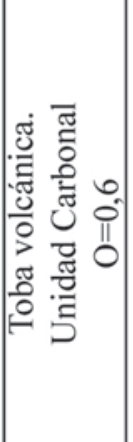 & 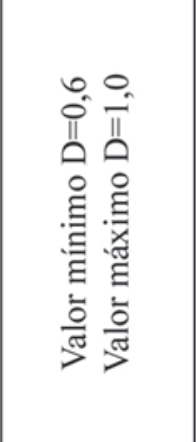 & 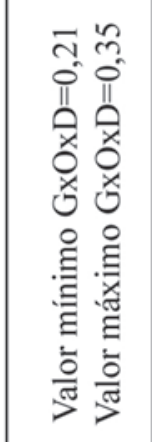 & 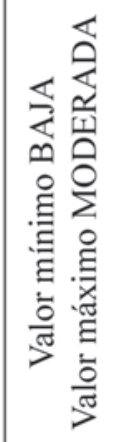 \\
\hline \multicolumn{5}{|c|}{$\begin{array}{l}\text { I.V.A. = G x O x D Índice de } \\
\text { vulnerabilidad acuífera CASO B }\end{array}$} \\
\hline $\begin{array}{l}\mathrm{G}^{*} \\
\text { Tipo } \\
\text { Acu- } \\
\text { ífero }\end{array}$ & $\begin{array}{c}\text { O* } \\
\text { Tipo de } \\
\text { cobe- } \\
\text { rtura } \\
\end{array}$ & $\begin{array}{c}\text { D** } \\
\text { Profundidad } \\
\text { a nivel de } \\
\text { saturación }\end{array}$ & GxOxD & $\begin{array}{l}\text { Clasifi- } \\
\text { cación }\end{array}$ \\
\hline 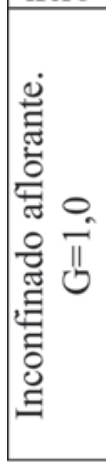 & 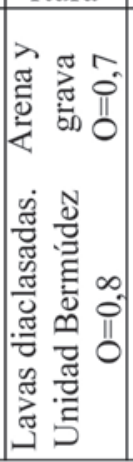 & 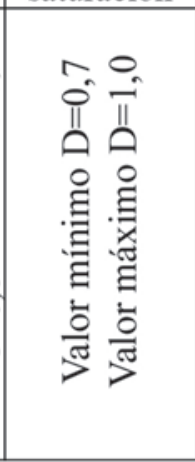 & 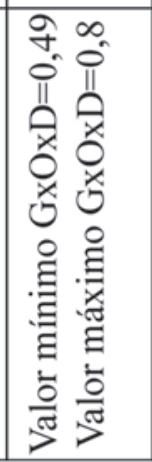 & 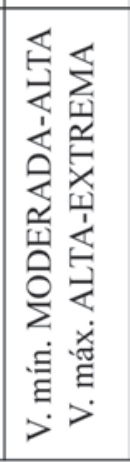 \\
\hline
\end{tabular}

*Información derivada de mapa hidrogeológico y datos estratigráficos de pozos

**Información derivada de datos de pozos, manantiales, ensayos geofísicos y perforación de piezómetros

Fig. 4: Caso A) Modelo de casos de vulnerabilidad a la contaminación acuífera específico para el cantón de Belén. Caso B) Modelo de casos de vulnerabilidad a la contaminación acuífera específico para el cantón de Belén.

baja tal sin que exista una transición al menos de vulnerabilidad moderada entre ambas.

Los parámetros de clasificación expuestos anteriormente resultan de la aplicación estricta y objetiva del método GOD, de manera que las investigaciones realizadas en este caso se orientaron a definir la distribución espacial (mapas) de los parámetros $\mathrm{G}$ y O mediante el levantamiento geológico de detalle y la revisión de la información estratigráfica de la figura 2, y del parámetro D mediante la determinación del nivel de saturación en el acuífero Barba en las zonas en donde dicho acuífero aflora en superficie.

\section{ZONIFICACIÓN DE PARÁMETROS QUE DEFINEN LA VULNERABILIDAD A LA CONTAMINACIÓN DEL ACUÍFERO BARBA EN EL CANTÓN DE BELÉN}

\section{Mapas geológico, hidrogeológico: Parámetros G y O}

La figura 6 muestra en mapa geológico hidrogeológico del cantón de Belén. Este mapa fue elaborado mediante observaciones y levantamientos de campo georeferenciados mediante GPS dentro del área que se muestra en la figura 6 .

Como se muestra, la mayor parte del sector Norte del cantón de Belén está compuesto por rocas y materiales correlacionados con la Formación Barba, específicamente por sus miembros Carbonal y Bermúdez (Protti, 1986).

Se trata de coladas de lavas de composición andesítica generadas en el macizo volcánico Barba, y que se extendieron irregularmente hasta a las inmediaciones de la ciudad de San Antonio. La expresión morfológica de los frentes terminales de estas coladas de lava se observa en los escarpes de rumbo general Este Oeste presentes entre la ciudad de San Antonio y La Ribera, así como entre La Asunción y Ciudad Cariari. Estas lavas afloran en forma dispersa a lo largo de su frente terminal, y en forma continua a lo largo de los cauces de los ríos Segundo y Burío en (Fig. $5 \mathrm{~B}$ ) donde la erosión ha penetrado a través de las capas de tobas de la unidad Carbonal.

La mayor parte del área en donde las unidades de lava del miembro Bermúdez están presentes en el subsuelo, muestra coberturas de tobas de ceniza alterada a limo arcillosos de baja permeabilidad que se correlacionan con el miembro Carbonal. Esta es la capa superior de coberturas 


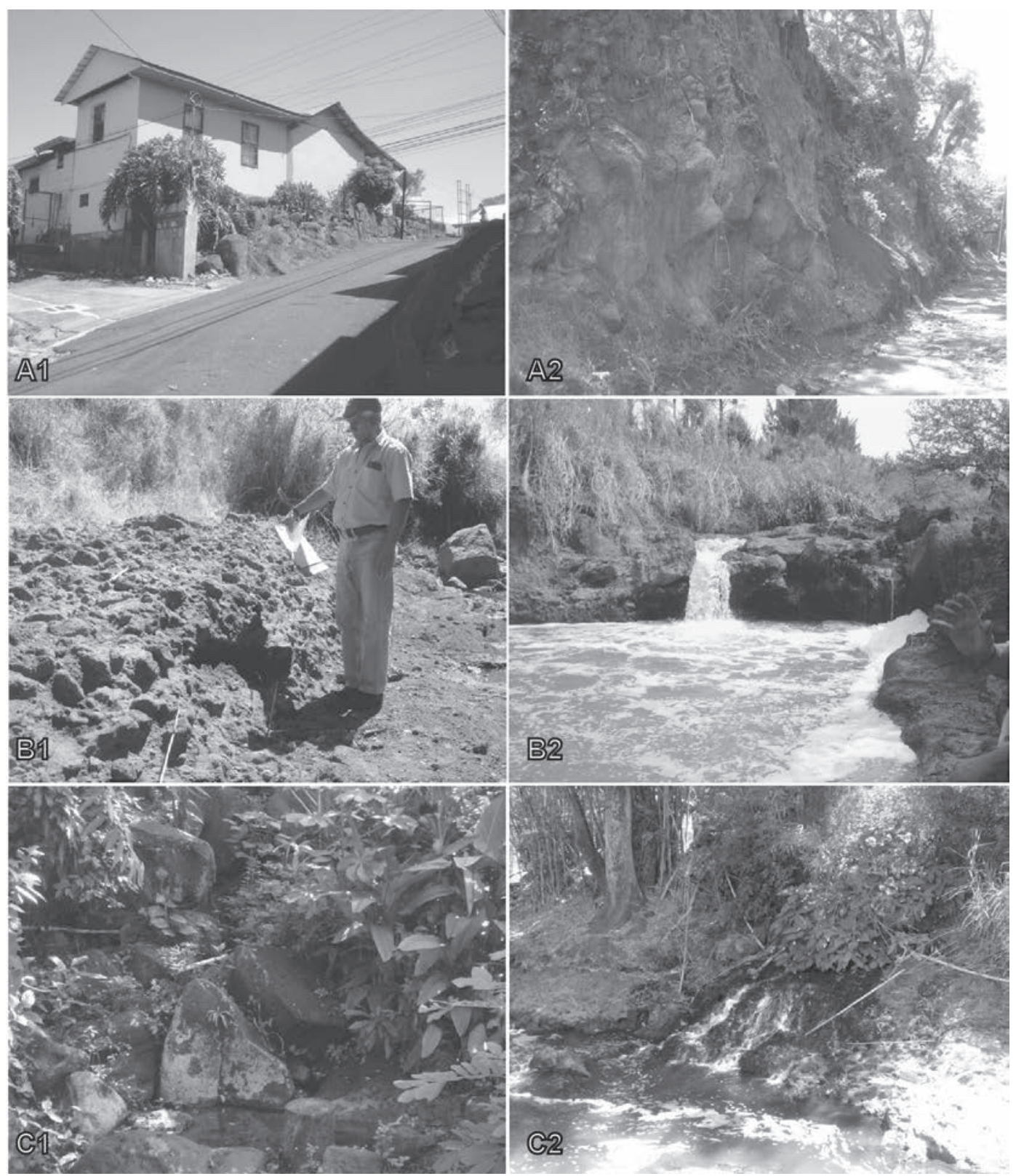

Fig. 5: A1 y A2) Afloramientos de las lavas de Bermúdez (acuífero Barba) sin coberturas de tobas. Calle Tilianos 218085-517380 (A1), Ojo de Agua, 218771-515253 (A2). B1) Brechas lávicas de Bermúdez en el cauce del río Burío (218353-516330). B2) Frente terminal de lavas de Bermúdez sobre ignimbritas de Tiribi, cauce de río Burío 218341-516627. C1) Manantial La Gruta (217683518010). C2 Manantiales en el cauce del río Burío (218422-517118).

sobre las lavas de Bermúdez, y su espesor medio es del orden de entre 10 y $20 \mathrm{~m}$.

Hacia la parte Sur del Cantón y hasta el borde y parte del talud del cañón del río Virilla, se presentan espesas coberturas de tobas e ignimbritas correlacionadas con la Formación Tiribí, las cuales cubren a las unidades de lava y brecha de Colima superior. Las rocas de la Formación 


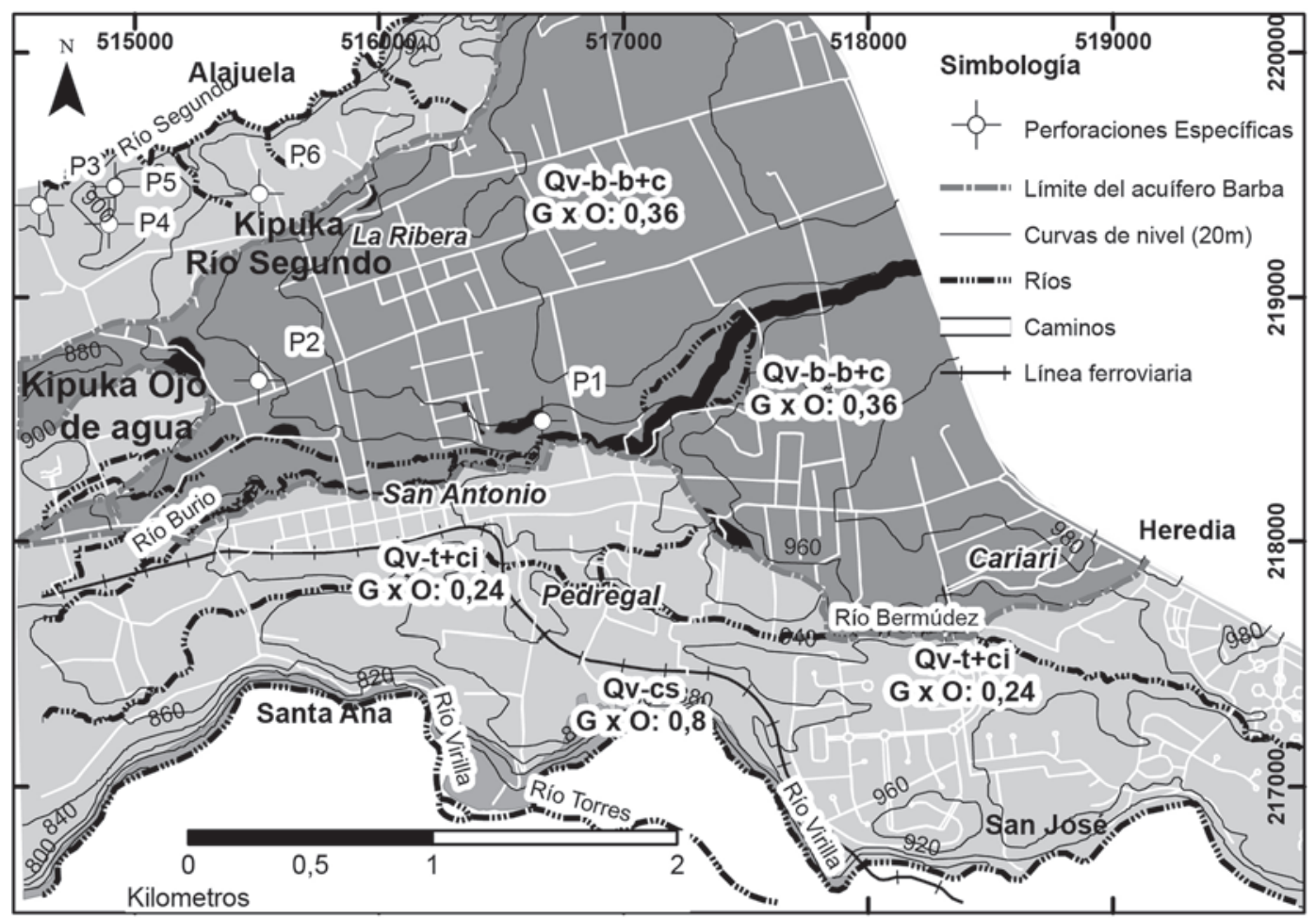

Geología-Hidrogeología

Qv-b-b+c

$\mathrm{G} \times \mathrm{O}=0,36$. Fm. Barba Unidad Bermúdez con coberturas de toba alterada de la Unidad Carbonal

Qv-bb

$\mathrm{G} \times \mathrm{O}=0,8$. Fm. Barba afloramiento de lavas. Unidad Bermúdez
Qv-t+ci

$\mathrm{G} \times \mathrm{O}=0,24$. Fm Tiribí. Toba ignimbrítica con coberturas indiferenciadas de toba alterada de la unidad Carbonal

\section{Qv-cs}

$\mathrm{G} \times \mathrm{O}=0,8$. Fm. Colima sup. Brecha y lava Unidad Belén

Fig. 6: Mapa geológico e hidrogeológico Mapa G x O.

Colima superior afloran a lo largo del cauce del río Virilla, en zonas dispersas dentro del cauce del río Bermúdez al este de San Antonio, y en el piso de canteras ubicadas cerca del cañón de dicho río (cantera Pedregal).

Hidrogeológicamente, las lavas de la unidad Bermúdez dan origen al acuífero Barba, el cual muestra condiciones de acuífero no confinado (Ramírez \& Alfaro, 2002), cubierto, con flujo de agua en dirección general Noreste a Suroeste, y cuya descarga natural ocurre en forma dispersa a lo largo de una serie de manantiales ubicados tanto en los frentes terminales de las lavas como en los bordes de cauces fluviales en donde las coberturas superficiales de tobas de Carbonal han sido erosionadas (Fig. 6).

En la figura 4 muestra la ubicación de las principales zonas de concentración de flujos de agua subterránea dentro del área investigada, la mayoría de las cuales representan las zonas en donde se produce la descarga natural de las aguas contenidas en el acuífero Barba.

Se observa claramente la tendencia de descargas en zonas de concentración de flujo a lo largo del frente terminal de las lavas de Barba, y en particular en la kipuka de Ojo de Agua, en 


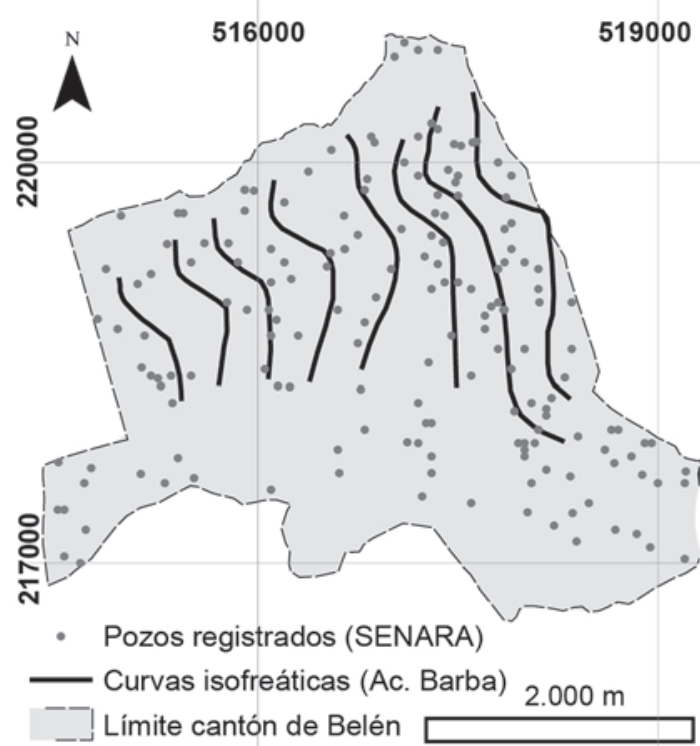

Fig. 7: Mapa de isofreáticas del acuífero Barba en el cantón de Belén. Fuente: Vargas (2009).

donde ocurre una de las mayores concentraciones de flujo de descarga desde dicho acuífero. Otras zonas importantes de descarga del acuífero Barba se localizan en los denominados manantiales $\mathrm{La}$ Gruta y Kimberly Clark, en Asunción, manantiales a lo largo del cauce del río Burío aguas arriba del plantel municipal (Fig. 7B y 7C), y la zona de manantiales Sánchez hacia el cauce del río Segundo en el extremo NE del área que se muestra en la figura 6.

Los datos e información hidrogeológica aportados por el levantamiento geológico hidrogeológico se utilizaron como base para la elaboración del mapa del producto entre los parámetros $\mathrm{G}$ y $\mathrm{O}$ del método utilizado para la evaluación de vulnerabilidad a la contaminación del acuífero Barba en el cantón de Belén, cuyo valor se indica en dicho mapa como el producto de las variables $\mathrm{G}$ x O (Fig. 6).

Se indica en dicho mapa tanto el tipo de acuífero presente en cada zona del área en donde el acuífero Barba está presente en el subsuelo (parámetro G), como el tipo de litología a través de la que ocurre percolación de aguas desde la superficie en la zona no saturada (parámetro O) hasta alcanzar el nivel de saturación. El resultado numérico del producto $\mathrm{G}$ x $\mathrm{O}$ se indica en el mapa de la figura 6 , según el método de análisis de vulnerabilidad aplicado en esta investigación.

De acuerdo con este mapa la mayor parte del área en donde se conoce con certeza la presencia de lavas de la unidad Bermúdez (acuífero Barba), las mismas se encuentran cubiertas por tobas alteradas. De esta forma, dichas áreas tienen valor $\mathrm{G}=0,6$ y $\mathrm{O}=0,6$, de manera que el producto $\mathrm{G} \times \mathrm{O}=0,36$.

Las zonas en donde el acuífero aflora en forma dispersa a lo largo de su frente terminal, y en forma continua a lo largo del cauce del río Burío, tienen valor $\mathrm{G}=1$ y $\mathrm{O}=0,8$ debido a que la percolación de aguas desde la superficie hasta el nivel de saturación ocurre a través de lavas diaclasadas (Método GOD, ver figura 1), y por lo tanto el producto $\mathrm{G}$ x $\mathrm{O}=0,8$.

Una vez definido el valor del producto $\mathrm{G}$ x $\mathrm{O}$, el resultado final del Índice de Vulnerabilidad Acuífera (IVA) depende de la variable D, es decir, de la profundidad al nivel de saturación en cada caso. De esta forma, en el capitulo siguiente se expone los resultados de la valoración de este parámetro.

\section{PROFUNDIDAD DEL NIVEL DE SATURACIÓN: PARÁMETRO D}

Para la definición del valor y sus variaciones del parámetro D (profundidad al nivel de saturación), se utilizó la información contenida en el informe realizado por la Universidad de Costa Rica para la delimitación de las zonas de protección de pozos del cantón de Belén (Vargas, 2009), específicamente el mapa de isofreáticas del acuífero Barba. (Fig. 7), y la información estratigráfica obtenida de las 6 perforaciones de piezómetros realizadas por Geotest S.A. entre mayo y setiembre de 2013, cuya ubicación se indica en las figuras 6 y 11.

En este mapa de isofreáticas (Vargas, 2009) (Fig. 7), se observa que la superficie freática del acuífero Barba en el cantón de Belén muestra una diferencia de elevación entre la equipotencial más baja y la más alta de $70 \mathrm{~m}(\mathrm{~V}=70 \mathrm{~m})$, en una distancia de $\mathrm{H}=3800 \mathrm{~m}$. 


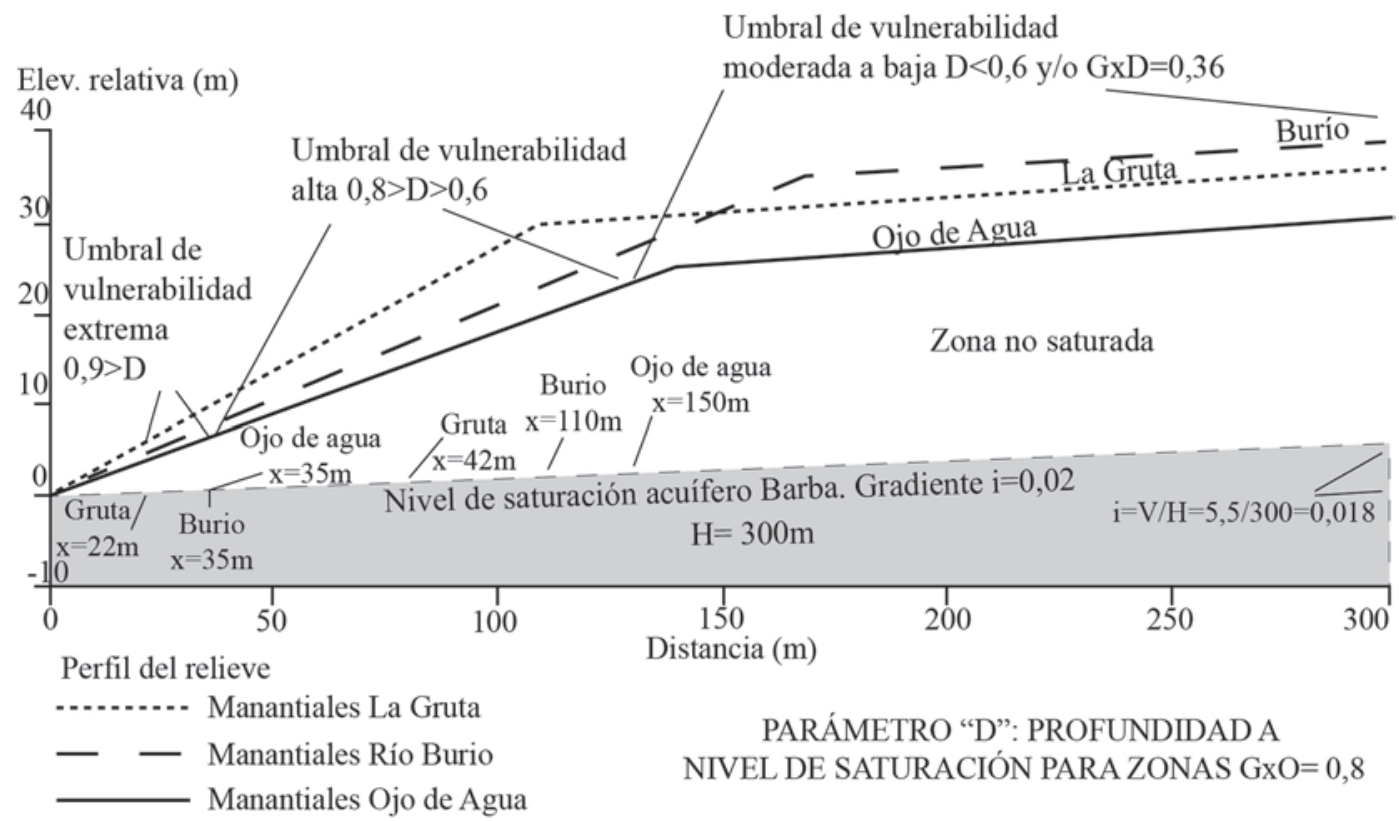

Fig. 8. Definición de parámetro D: profundidad al nivel de saturación.

De esta forma, el gradiente hidráulico (i) resultante para el acuífero Barba en el área que se muestra en la figura 6 es de:

$\mathrm{I}=\mathrm{V} / \mathrm{H}=70 / 3800=\mathrm{i}=0,018$.

Así, el valor del parámetro D (profundidad al nivel de saturación) aguas arriba de las zonas en donde el acuífero se descarga naturalmente (manantiales en donde $\mathrm{H}=0$ ), se obtuvo para cada caso mediante la superposición de las curvas de relieve superficial del terreno y de la superficie freática congruente con el gradiente hidráulico del acuífero, en este caso se utilizó un valor de gradiente de $\mathrm{i}=0,02$ que contiene un factor de seguridad de $\mathrm{Fs}=0,02 / 0,018=1,1$.

Lo anterior se aplicó para los casos específicos en donde el valor de la profundidad al nivel de saturación es crítico para diferenciar las zonas de vulnerabilidad extrema y alta, es decir, para aquellos casos donde el producto de las variables $\mathrm{G} \times \mathrm{O}=0,8$.

Este caso aplica para las zonas en donde las lavas de la Unidad Bermúdez están expuestas en superficie (acuífero no confiado $G=1$ ), y la percolación de aguas en la zona no saturada ocurre a través de dichas lavas $(\mathrm{O}=0,8)$.

La figura 8 muestra los casos analizados para las secciones correspondientes a los manantiales de La Gruta, manantiales a lo largo del cauce del río Burío aguas arriba del plantel municipal, y para los manantiales de Ojo de Agua. Esta figura muestra el perfil del relieve obtenido de la sección topográfica del mapa a escala 1:10 000 de los 3 casos tipo, y su relación con la superficie freática del acuífero Barba.

La gráfica de la figura 8 permite definir la distancia aguas arriba desde las zonas de descarga $(\mathrm{H}=0)$ hasta los límites o umbrales en donde los valores del parámetro $\mathrm{D}$ determinan si el resultado del producto $\mathrm{G} \times \mathrm{O}$ x D corresponde con zonas de vulnerabilidad extrema ( $\mathrm{H}<5 \mathrm{~m}$ ) o alta ( $5 \mathrm{~m}$ $<\mathrm{H}<20 \mathrm{~m}$ ) para las áreas en donde el acuífero se encuentra expuesto en superficie, es decir, con $\mathrm{G} \times \mathrm{O}=0$,8. Los resultados de este análisis se muestran en el cuadro 1.

El cuadro 2 muestra los resultados obtenidos en las 6 perforaciones realizadas en las áreas 

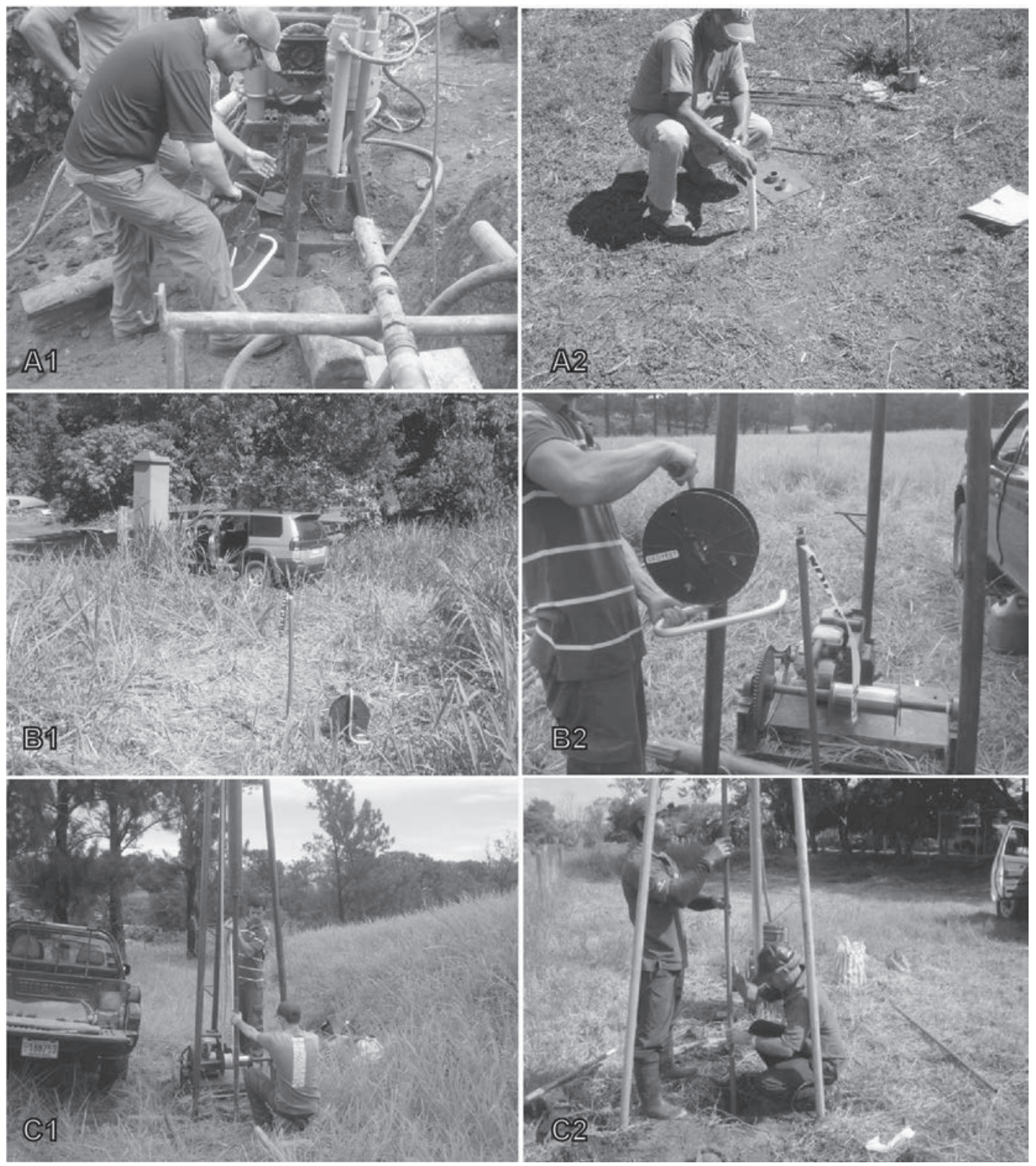

Fig. 9: A1 y A2) Fotografías: piezómetros P1 y P2. B1 y B2) Piezómetros P3 y P4. C1 y C2) Piezómetros P5 y P6 respectivamente.

clasificadas como de extrema vulnerabilidad en el mapa oficial en donde se observa que dichos resultados difieren totalmente de los parámetros necesarios para clasificar estas áreas como de extrema vulnerabilidad, es decir, la única forma posible de clasificar como extrema vulnerabilidad a cualquier área en el cantón de Belén es aquella en que el acuífero Barba de tipo no confinado aflore en superficie $(\mathrm{G}=1)$, que la percolación de aguas desde la superficie ocurra a través de lavas "recientes" $(\mathrm{O}=0,8)$, y que el nivel de saturación se ubique a menos de $5 \mathrm{~m}$ de profundidad $(\mathrm{D}=0,8)$, es decir, para IVA $=1 \times 0,8 \times 0,9=0,72$, y esta condición no ocurre en ninguno de los sitios investigados directamente mediante la perforación de piezómetros. 


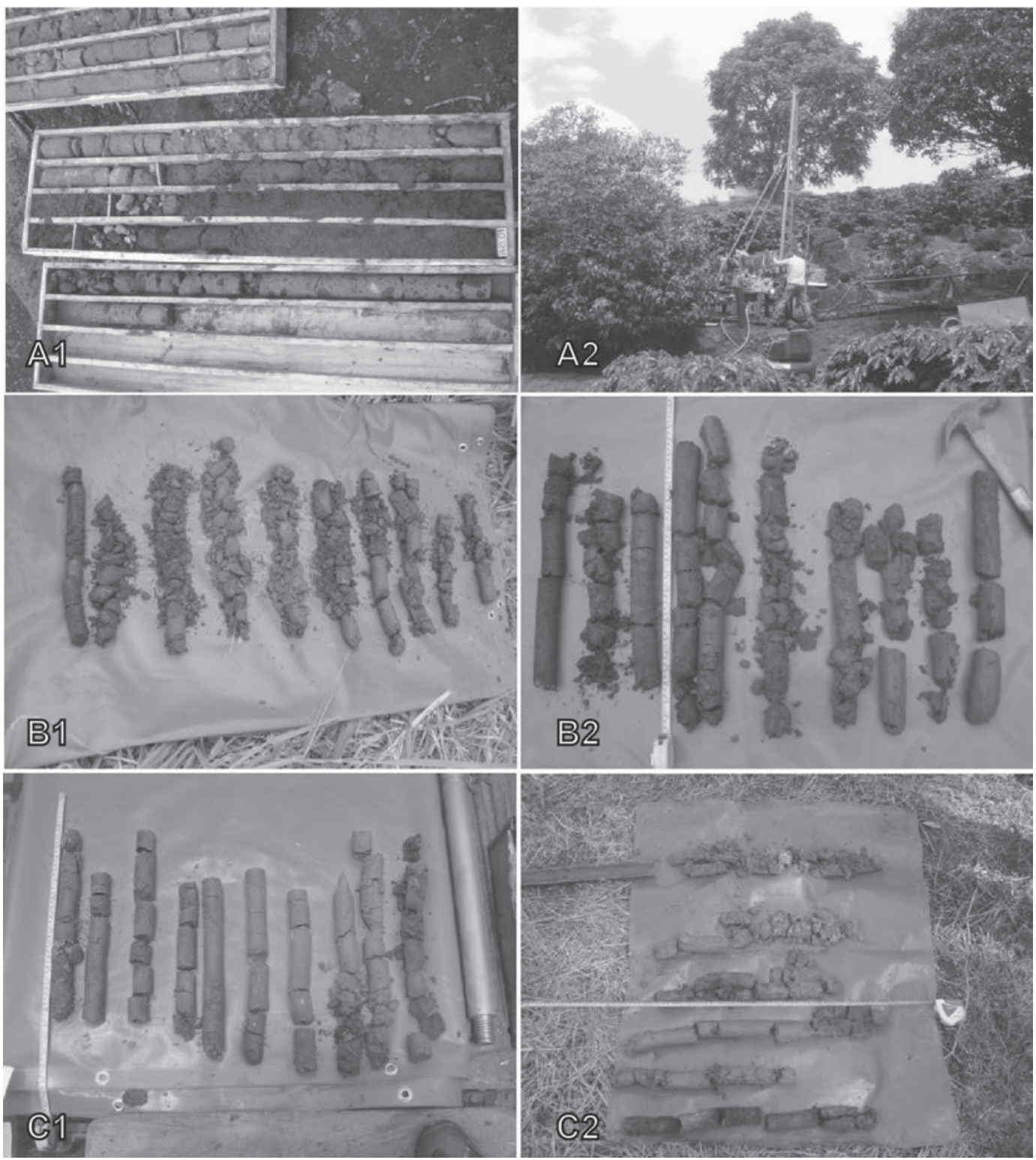

Fig. 10: A1 y A2) Materiales arcillo arenosos no saturados con ignimbrita (Tiribi) como basamento en la perforación P1 ubicada en el frente lateral de la Fm. Barba, sobre la margen derecha del río Burío. B1 y B2) Alteraciones arcillosas de toba obtenidas en las perforaciones P3 y P4, ambas sobre laderas de la margen izquierda del río Segundo. C1 y C2) Alteraciones arcillosas de tobas obtenidas en las perforaciones P5 y P6.

La información derivada del modelo de la figura 8 , del cuadro 1 (modelo de distancias críticas por gradiente), y del cuadro 2 (datos de perforaciones realizadas para esta investigación), se aplicó como variable al mapa de producto $\mathrm{G} x$ O para determinar numéricamente la extensión de las zonas de vulnerabilidad extrema (IVA $>$ $0,7)$ y de vulnerabilidad alta $(0,5<$ IVA $<0,7)$ para aquellas zonas en donde las lavas de la unidad Bermúdez afloran en superficie y que además constituyan zonas de descarga del acuífero Barba, es decir, para las zonas en donde $\mathrm{H}=0$. 
Cuadro 1

Distancia de umbral de clasificación

\begin{tabular}{ccc}
\hline Sitio tipo & $\begin{array}{c}\text { Distancia EX- } \\
\text { TREMAH }<5 \mathrm{~m}\end{array}$ & $\begin{array}{c}\text { Distancia ALTA5 } \\
\mathrm{m}<\mathrm{H}<20 \mathrm{~m}\end{array}$ \\
\hline La Gruta & $22 \mathrm{~m}$ & $80 \mathrm{~m}$ \\
Burío & $30 \mathrm{~m}$ & $110 \mathrm{~m}$ \\
Ojo de Agua & $35 \mathrm{~m}$ & $130 \mathrm{~m}$ \\
\hline
\end{tabular}

Asimismo, el límite entre las zonas de vulnerabilidad moderada y vulnerabilidad baja está definido por la ubicación o límite entre las zonas en donde el acuífero Barba está cubierto $(\mathrm{G} \times \mathrm{O}=$ $0,36)$, y las zonas en donde el frente de las lavas está expuesto $(\mathrm{G} \times \mathrm{O}=0,8)$ pero el nivel de saturación se localiza a más de $20 \mathrm{~m}$ de profundidad.

De esta forma, cada zona de vulnerabilidad es envolvente con respecto a la zona siguiente de mayor vulnerabilidad, lo cual es consecuente con el modelo hidrogeológico del cantón, en donde las variaciones tanto de la superficie de saturación como del relieve del terreno son leves y transicionales.

Todas las perforaciones realizadas (Fig. 9) para esta investigación fueron ubicadas en zonas clasificadas como de vulnerabilidad extrema en el mapa oficial sin embargo, los resultados obtenidos no concuerdan en forma alguna con dicha clasificación.

Estos sondeos no detectaron presencia de lavas ni nivel de saturación a menos de $5 \mathrm{~m}$ de profundidad, factor que es determinante en la previa clasificación de estos sitios como de extrema vulnerabilidad.
Las figura 10 muestra el tipo de materiales obtenidos en las perforaciones, con excepción de la P2, la cual se realizó mediante penetración de cono dinámico. La ubicación de las perforaciones se muestra en las figuras 6 (mapa hidrogeológico $\mathrm{GxO}$ ) y 18 (mapa de vulnerabilidad).

El resultado final de la superposición de las variables $\mathrm{G}$ x O x D es el mapa de vulnerabilidad a la contaminación del acuífero Barba en el cantón de Belén que se describe en el capítulo siguiente.

\section{MAPA DE VULNERABILIDAD A LA CONTAMINACIÓN ACUÍFERA EN EL CANTÓN DE BELÉN}

La figura 11 muestra el mapa final y específico de vulnerabilidad a la contaminación acuífera dentro del cantón de Belén. Como se muestra, la superposición de los parámetros G x O x D y su resultado en términos del Índice de Vulnerabilidad Acuífera (IVA) permite definir las zonas de vulnerabilidad que se describen a continuación.

Zona de vulnerabilidad BAJA (IVA $<0,3$ ): Comprende aquellas zonas en donde el acuífero Barba de tipo no confinado (Unidad Bermúdez) se encuentra cubierto por tobas alteradas (Unidad Carbonal), con parámetro $\mathrm{G}=0,6$ y $\mathrm{O}=0,6$. En este caso, el índice de vulnerabilidad a la contaminación acuífera es IVA $<0,36$. En todos los casos en donde el nivel de saturación se localice a más de $5 \mathrm{~m}$ de profundidad, es decir, con $\mathrm{G}=0,8$, el valor IVA $=0,6 \times 0,6 \times 0,8=0,28$.

Cuadro 2

Datos básicos de piezómetros perforados durante la investigación

\begin{tabular}{cccccc}
\hline Perf. No. & X & Y & Prof. $(\mathrm{m})$ & N.S. (m.b.n.s.) & Litología \\
\hline P1 & 516665 & 218494 & 12 & $>10$ & Aluvial fino sobre tobas Tiribi \\
P2 & 515504 & 218657 & 5 & $>5$ & Arcilla residual \\
P3 & 514588 & 219376 & 6 & $>6$ & Toba alterada a arcilla plástica \\
P4 & 514893 & 219299 & 6 & $>6$ & Toba alterada a arcilla plástica \\
P5 & 514919 & 219452 & 6 & $>6$ & Toba alterada a arcilla plástica \\
P6 & 515506 & 219423 & 6 & $>6$ & Toba alterada a arcilla plástica \\
\hline
\end{tabular}




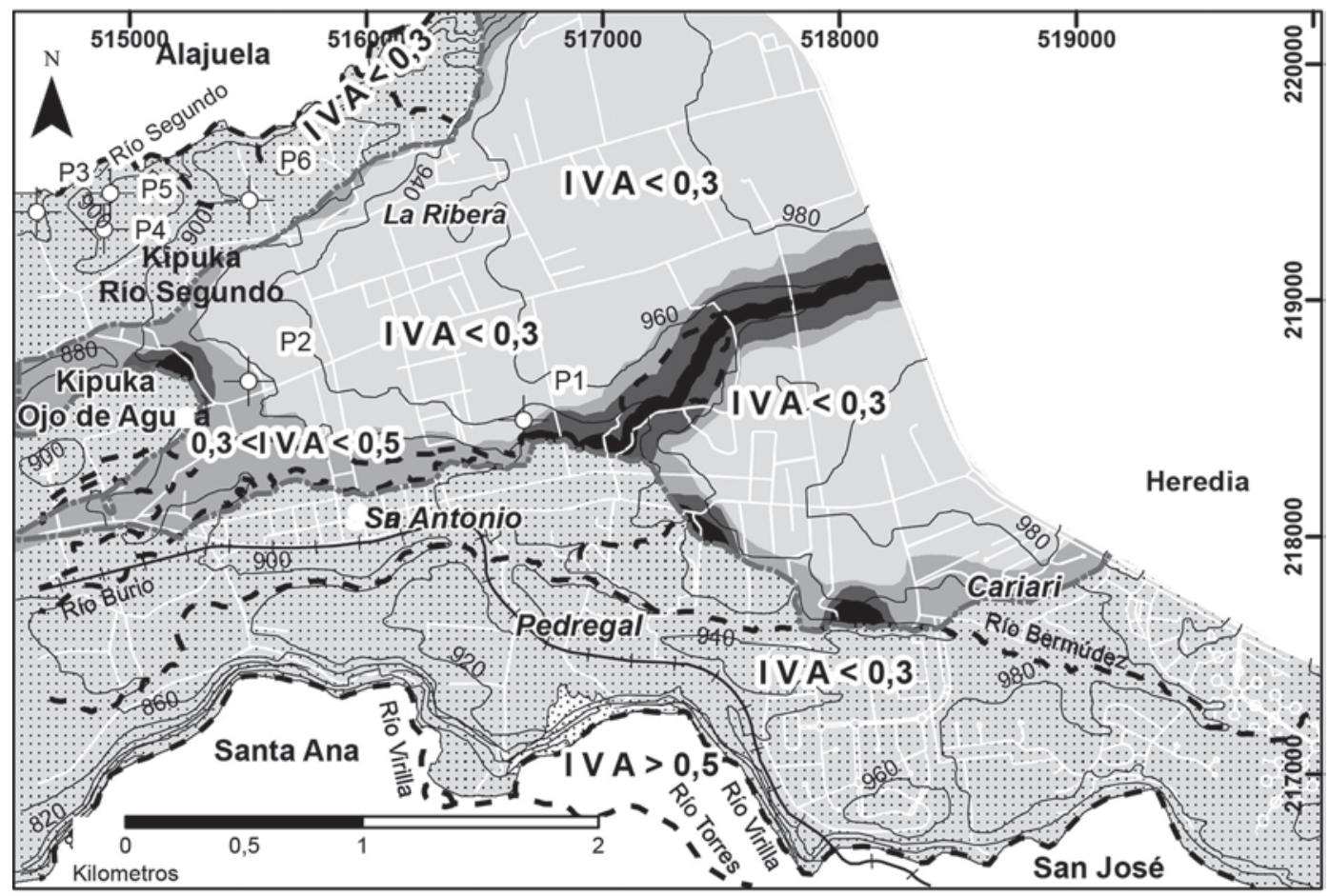

Simbología

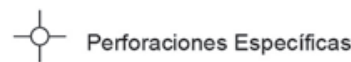

-_- Límite del acuifero Barba

Curvas de nivel $(20 \mathrm{~m})$

Caminos

- Ríos

$\longrightarrow$ Línea ferroviaria
I.V.A.

Acuífero Colima Acuífero Barba

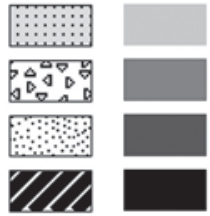

Baja vulnerabilidad IVA $<0,3$

Mod. vulnerabilidad $0,3<$ IVA $<0,5$

Alta vulnerabilidad $0,5<$ IVA $<0,7$

Extrema vulnerabilidad $0,7<$ IVA

Fig. 11: Mapa de vulnerabilidad a la contaminación acuífera en el cantón de Belén.

Dentro de esta categoría se incluyen las áreas del sector Sur del cantón en donde el acuífero de Colima superior se encuentra cubierto por espesas capas de tobas de la unidad Tiribi.

Zona de vulnerabilidad MODERADA $(0,3$ $<$ IVA $<0,5)$ : Comprende las áreas en donde el acuífero Barba se encuentra cubierto por tobas alteradas de la unidad Carbonal ( $\mathrm{G}$ x $\mathrm{O}=0,36)$, y el nivel de saturación se ubica a menos de $5 \mathrm{~m}$ de profundidad $(\mathrm{D}=0,9-1,0)$. En estos casos, el valor de IVA varía entre 0,32 y 0,36 , que corresponde con zonas de vulnerabilidad moderada. La resolución de esta clasificación no es muy alta, es decir, las incertidumbres inherentes a este tipo de investigación (Foster et al., 2002) implican que es altamente probable que la mayor parte de las áreas clasificadas dentro de esta categoría correspondan más bien con zonas de baja vulnerabilidad.

Las áreas clasificadas como de vulnerabilidad moderada cubren la zona de transición entre las áreas de baja y alta vulnerabilidad, y su extensión o ancho es variable según el perfil del relieve del terreno con respecto a la superficie de saturación. 
Zona de vulnerabilidad ALTA $(0,5<$ IVA $<0,7)$ : Comprende aquellas área en donde el acuífero Barba aflora en superficie $(\mathrm{G} \times \mathrm{O}=0,8)$ o se encuentra cubierto por capas de sedimentos aluviales finos ( $\mathrm{G} \times \mathrm{O}=0,7)$, y en donde el nivel de saturación se localiza a más de $5 \mathrm{~m}$ de profundidad $(\mathrm{D}=0,8)$ pero a menos de $10 \mathrm{~m}(\mathrm{D}$ $=0,7)$. En ambos casos el valor IVA es de $0,8 \mathrm{x}$ $0,8=0,64$ y $0,8 \times 0,7=0,56$, respectivamente, es decir dentro del rango que caracteriza como de alta vulnerabilidad según el método GOD. (Rango de alta vulnerabilidad IVA=0,5-0,7).

Según se muestra en el mapa de la figura 11, zonas de vulnerabilidad alta solo pueden ser definidas numéricamente en la periferia de las zonas de extrema vulnerabilidad que se describen en el siguiente apartado.

Zonas de vulnerabilidad alta también se determinan numéricamente para las áreas de canteras junto al cauce del río Virilla en donde el acuífero de Colima superior se encuentra expuesto aguas arriba de los manantiales de Puente de Mulas. Las áreas de vulnerabilidad alta son envolventes con respecto a las zonas de vulnerabilidad extrema.

Zona de vulnerabilidad EXTREMA (IVA > 0,7). Estas zonas son determinadas para los sitios o áreas en donde se produce superficialmente la descarga de aguas desde el acuífero Barba, es decir, en zonas de concentraciones de flujo o manantiales, específicamente para las áreas circundantes de los manantiales La Gruta, Kimberly Clark, manantiales a lo largo del cauce del río Burío aguas arriba del plantel municipal, Ojo de Agua y Sánchez y similares a lo largo de la margen izquierda del río Segundo.

El ancho o distancia determinante de las zonas de vulnerabilidad extrema fueron definidos en el cuadro A de este informe sobre la base de la sustracción entre el relieve de superficie y la gradiente de la superficie de saturación, y verificados mediante la perforación de 6 piezómetros en sitios críticos seleccionados que fueron previamente clasificados como de extrema vulnerabilidad en el mapa oficial.

Sin excepción, los resultados de las perforaciones y sondeos realizados específicamente para esta investigación indican que en los sitios sondeados no se cumple en forma alguna ninguno de los requisitos necesarios para que una zona sea clasificada como de vulnerabilidad extrema en las condiciones hidrogeológicas del cantón de Belén, es decir, presencia de afloramientos de un acuífero no confinado $(G=1)$, percolación a través de lavas $(\mathrm{O}=0,8)$, y nivel de saturación a menos de 5 $m$ de profundidad $(D=0,9)$. Cualquier condición diferente a esta en el cantón de Belén no puede ser clasificada como de vulnerabilidad extrema.

\section{CONCLUSIONES}

Con base en lo expuesto en este informe, y sobre todo, sobre la base de los aportes inéditos de esta investigación en términos de la elaboración de un mapa detallado de los parámetros $\mathrm{G}$ x O y verificación in situ mediante la perforación de 6 sondeos estratigráficos y piezométricos, se concluye que:

- La aplicación rigurosa y objetiva del método G.O.D. para la elaboración del mapa de vulnerabilidad a la contaminación acuífera en el cantón de Belén de Heredia, conduce a la definición de 4 zonas de vulnerabilidad a la contaminación que varían entre BAJA, MODERADA, ALTA y EXTREMA según las condiciones locales.

- La mayor parte de los terrenos estudiados se ubican en zonas de BAJA y MODERADA vulnerabilidad, mientras que zonas de vulnerabilidad ALTA y EXTREMA solo es posible determinarlas, mediante análisis numérico y perforación de piezómetros estratigráficos, únicamente para las áreas circundantes a las zonas de descarga natural del acuífero Barba, es decir, para las zonas ubicadas gradiente arriba de los sitios de manantiales, a la distancia crítica determinada por la sustracción entre las cotas del perfil topográfico del terreno y las cotas de la superficie de saturación dentro del acuífero, y verificadas con al menos 6 sondeos exploratorios.

- De acuerdo con el método GOD, para las condiciones hidrogeológicas del cantón de Belén, solo es posible clasificar numéricamente como zonas de extrema vulnerabilidad a la contaminación a zonas con afloramientos de un acuífero volcánico no confinado $(G=1)$, con percolación a través de lavas $(\mathrm{O}=0,8)$, y nivel de saturación a 
menos de $5 \mathrm{~m}$ de profundidad $(\mathrm{D}=0,9)$. Esta condición no se cumple en al menos el 90 \% de la zona que fue clasificada dentro de esta categoría en el mapa oficial.

- El mapa de vulnerabilidad generado durante esta investigación (Fig. 11), difiere sensiblemente del mapa de vulnerabilidad a la contaminación acuífera actualmente en vigencia por parte de la Municipalidad de Belén.

\section{REFERENCIAS BIBLIOGRÁFICAS}

AUGE, M., 2004. Vulnerabilidad de acuíferos: Conceptos y métodos.- 84 págs. Ed. Universidad de Buenos Aires, Buenos Aires.

FOSTER, S., HIRATA, R., GOMES, D., D ELIA, M., PARIS, M., 2002: Protección de la calidad del agua subterránea: guía para empresas de agua, autoridades municipales y agencias ambientales.- 115 págs. Banco Mundial, Berlín.
PÉREZ, W., ALVARADO, G. E. \& GANS, P. B., 2006. The 322 Ka Tiribí Tuff: Stratigraphy, Geochronology and Mechanism of Deposition of the Largest and Most Recent Ignimbrite in the Valley Central, Costa Rica.- Bull. Volcanol. 69: 25-40.

PROTTI, R. 1986. Geología del flanco sur del volcán Barba.- Bol. Vulcanol. Univ. Nac. 17: 23-31.

RAMÍREZ, R. 2002. Mapa de vulnerabilidad hidrogeológica de una parte del valle Central de Costa Rica.- Rev. Geol. Amér. Central, 27: 56-60.

BGS \& SENARA, 1985: Mapa hidrogeológico del valle Central, Costa Rica.- Escala 1:50 000, E.S.R. Limited, Inglaterra.

VARGAS, I., 2009. Estudio para la delimitación de las zonas de protección de los pozos para abastecimiento público, Municipalidad de Belén.- 70 págs. UCR-Senara-Municipalidad de Belén, San José [Inf. interno]. 